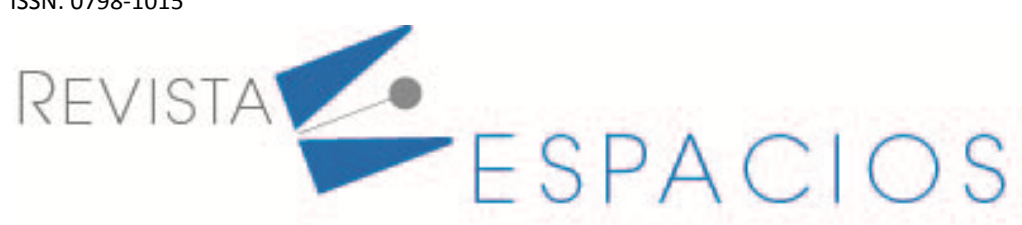

\title{
Caracterización de lineamientos de accesibilidad para plataformas educativas con énfasis en contenido de video
}

\author{
Characterization of accessibility guidelines for educational platforms with emphasis on \\ video content
}

\author{
PÉREZ, Daniela. ${ }^{1}$ \\ RUIZ, Alexandra. ${ }^{2}$ \\ CHANCHÍ, Gabriel ${ }^{3}$
}

\section{Resumen}

Se realizó una revisión sistemática entorno a propuestas enfocadas en lineamientos de accesibilidad para plataformas educativas basadas en contenidos de video. Dichas propuestas fueron clasificadas de acuerdo con diferentes aspectos. La clasificación permitió identificar fortalezas y brechas para suplir en trabajos futuros. Dentro de las brechas están: escasas propuestas enfocadas hacia la discapacidad motriz o intelectual, bajo formalismo y poca documentación sobre la implementación y evaluación de cada lineamiento.

Palabras clave: accesibilidad, discapacidad, educación virtual, lineamientos de accebilidad, multimedia.

\begin{abstract}
A systematic review was conducted regarding proposals focused on accessibility guidelines for educational platforms based on video content. These proposals were classified according to different aspects. The classification allowed the identification of strengths and gaps to be filled in future work. Among the gaps are: few proposals focused on motor or intellectual disability, low formalism and little documentation on the implementation and evaluation of each guideline.

Key words: accessibility, accessibility guidelines, disability, multimedia, virtual education.
\end{abstract}

\section{Introducción}

El uso de tutoriales audiovisuales de corta duración constituye un recurso formativo de gran atractivo para los jóvenes estudiantes, quienes se han familiarizado con este tipo de formato similar al utilizado en YouTube, es por esto que diferentes autores los han denominado como píldoras formativas o educativas (Muñoz Cantero, Espiñeira-Bellón, and Rebollo-Quintela 2016). Se trata de una pequeña pieza de contenido audiovisual diseñada para complementar las estrategias tradicionales de formación, sin embargo, este tipo de material multimedia, a

\footnotetext{
${ }^{1}$ Ingeniera Informatica del Colegio Mayor del Cauca. Estudiante de Msc en Ingeniera de Software de la Universidad del Quindio. Sus áreas de interés el $\mathrm{HCl}$, Accesibilidad y Diseño de interfaces. daniela.perezo@uqvirtual.edu.co

2 PhD en Ingeniería Telemática de la Universidad del Cauca. Profesora de tiempo completo en la Universidad Quindío. Programa de Ingería de Sistemas y Computación. Sus áreas de interés son la ingeniería de software y $\mathrm{HCl}$. aruiz@uniquindio.edu.co

3 Profesor de la Universidad de Cartagena. Programa de Ingeniería de Sistemas. Facultad de Ingeniería. Ingeniero en Electrónica y Telecomunicaciones, MSc. en Ingeniería Telemática, PhD. en Ingeniería Telemática.gchanchig@unicartagena.edu.co
} 
diferencia de otros materiales didácticos clásicos, evidencia problemas de usabilidad y accesibilidad al ser utilizados por estudiantes con alguna discapacidad (García Pérez and Ortega Sánchez 2010). En los últimos años el desarrollo de las tecnologías de la información y el conocimiento han tenido un notable impacto en la enseñanza universitaria a distancia, tanto a nivel cuantitativo como cualitativo. Se ha observado que a pesar de la aparición de numerosas y novedosos recursos didácticos, el video, en su forma convencional o incorporando la interactividad, sigue siendo todavía el preferido por los profesores y alumnos para mejorar la calidad del proceso de aprendizaje (De la Fuente Sánchez et al., 2017).

Nadie duda que la evolución de la tecnología ha provocado cambios sustanciales en las sociedades en las que se ha desarrollado. En este contexto de continuas modificaciones, las tecnologías de la información y la comunicación (TIC) no han sido ajenas a estos fenómenos de transformación en diversos ámbitos, incluido por supuesto, el de la educación y el aprendizaje (Belloch 2012). Muchos autores han señalado a lo largo de los últimos años las ventajas de las TIC en los entornos de enseñanza y aprendizaje, tales como: la ampliación de la oferta formativa e informativa, la apertura de nuevas posibilidades de la tutorización, la eliminación de barreras de espacio y de tiempo, la facilitación del trabajo personal, del trabajo colaborativo y del autoaprendizaje, además de potenciar la interactividad y la flexibilidad en el aprendizaje (De la Fuente Sánchez et al., 2017), (Hernández, 2017).

En esta evolución hacia la docencia en línea o educación digital ha sido fundamental el desarrollo de internet y su capacidad para integrar un conjunto de materiales multimedia de diversa naturaleza (webconferencias, videoconferencias, chats, videos y audios, minilibros electrónicos o laboratorios remotos). Este tipo de materiales debería tener un mínimo nivel de accesibilidad que permita minimizar la brecha de exclusión por parte de plataformas educativas para personas con algún tipo de discapacidad (Hernández 2013). Teniendo en cuenta este contexto, este artículo tiene como propósito caracterizar propuestas en el marco de los lineamientos de accesibilidad para portales educativos con énfasis en contenido de video de acuerdo con diferentes enfoques. A partir de la caracterización se evidencian las fortalezas de las propuestas evaluadas y posibles brechas que puedan dar inicio a futuras investigaciones, en términos de la formulación de lineamientos de accesibilidad para plataformas educativas soportadas en video.

El presente artículo se encuentra estructurado de la siguiente manera: en la sección 2 se presenta la metodología de trabajo, en la cual se aborda el método de caracterización para realizar la revisión sistemática de las propuestas y la clasificación de las propuestas seleccionadas; en la sección 3 se presenta el desarrollo realizado para la revisión sistemática, el cual describe las propuestas seleccionadas y la clasificación de dichas propuestas de acuerdo con los diferentes criterios relacionados a las preguntas de investigación; la sección 4 presenta una discusión referente a los hallazgos más importantes y, finalmente, la sección 5 expone las conclusiones y trabajos futuros derivados de la presente investigación.

\section{Metodología de trabajo}

En esta sección se presenta el método que guía la caracterización de propuestas que se evidencian en la literatura relacionadas con lineamientos de accesibilidad para el diseño de portales educativos con énfasis en contenido de video (ver Figura 1).

Las propuestas analizadas se sitúan dentro del dominio de la accesibilidad, específicamente aquellas que se aplican en plataformas de aprendizaje en línea con énfasis en video (subdominio) y en el contexto de $\mathrm{HCl}$. Una vez identificado el dominio y el contexto se procede a realizar una revisión sistemática, la cual fue concebida de acuerdo con el proceso descrito por Kitchenham (Ruiz, Arciniegas, and Giraldo 2018). La revisión sistemática tiene el fin de hacer una búsqueda rigurosa y formal frente a heurísticas, pautas o guías de accesibilidad para el diseño de interfaces con contenido de video en plataformas educativas. A partir de la revisión sistemática se 
pueden evidenciar las propuestas de lineamientos de accesibilidad plateados por diferentes autores y las fortalezas y brechas encontradas en dichas propuestas.

Figura 1

Método de caracterización

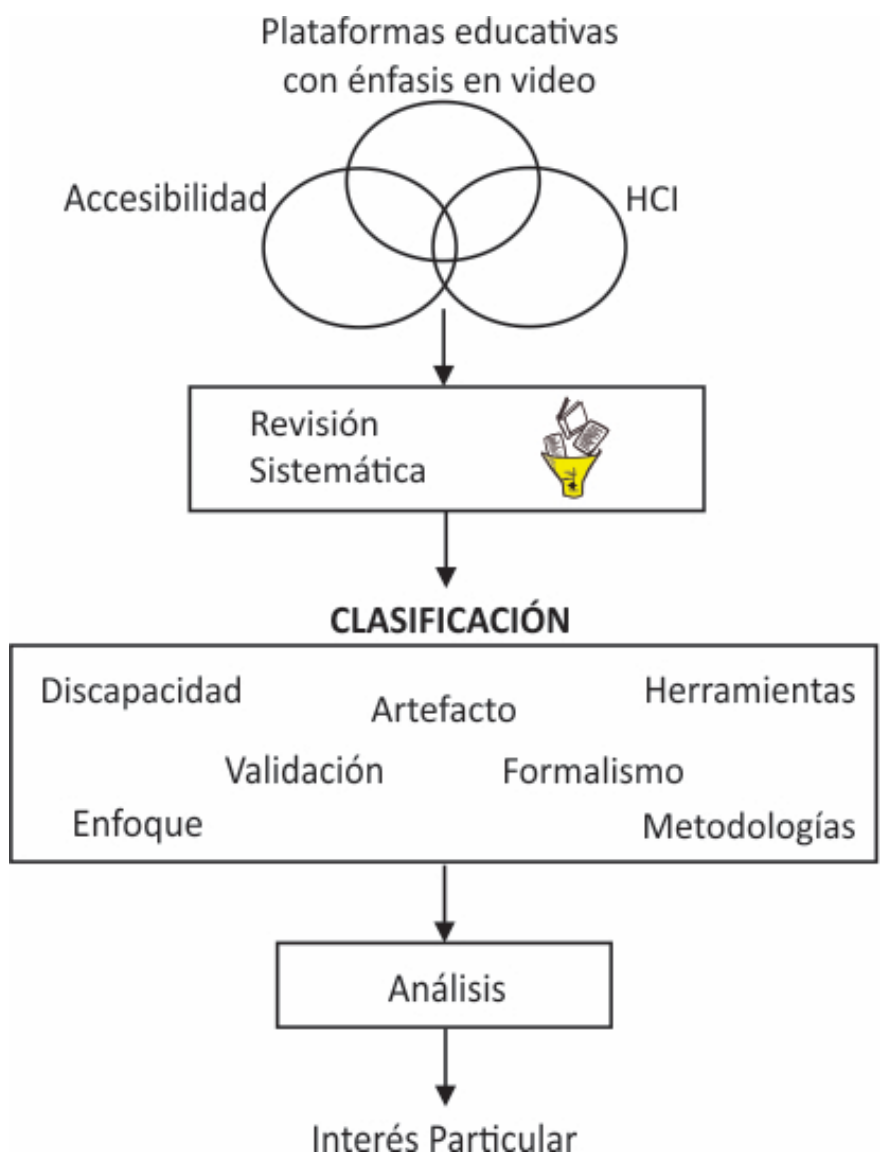

Fuente: Elaboración propia

A continuación se presenta el diseño de la revisión, el cual consta de las siguientes secciones:

Preguntas de investigación: La revisión estuvo orientada a responder las siguientes preguntas de investigación:

- P1: ¿Qué artefactos evidencia la literatura para promover la accesibilidad en los diversos sistemas interactivos y qué tipo de enfoques utilizan estos estudios?

- P2: ¿Qué nivel de formalismo y tipo de validación tienen las propuestas evaluadas?

- P3: ¿Qué herramientas que promuevan la accesibilidad son resultados de las propuestas evaluadas?

Enfoque de búsqueda: El enfoque de búsqueda estuvo orientado a identificar estudios previamente realizados en publicaciones como revistas, libros, eventos o ponencias donde se pudiera identificar propuestas de valor en el diseño de interfaces con contenido de video en plataformas educativas con énfasis accesibilidad. La cadena de búsqueda utilizada para identificar estos estudios primarios se presenta a continuación:

- C1 (Qué)=("Norms" OR "Guide" OR "Standards"OR "Guidelines"OR "Techique") AND("engineerieng " OR "Model" OR "Methodology").

- C2 (Dónde)=("Video" OR "Multimedia") AND ("Educational" OR "Platforms"). 
- C3 (Integra)=("Accessibility" OR "Usability"OR "Learning").

De esta manera la cadena completa usada en la búsqueda fue: C1 AND C2 AND C3

Selección de estudios: A continuación se presentan los criterios que se tuvieron en cuenta para seleccionar las propuestas a evaluar:

- Estudios relacionados con ciencias de la computación o ingeniera de software y el aprendizaje a distancia, virtual o b-learning.

- Estudios relacionados con la implementación de técnicas de diseño de interfaces con contenido de video en plataformas de aprendizaje.

- Estudios que integren una metodología de accesibilidad y aprendizaje a distancia.

- Estudios que se realicen desde el 2010 hasta la fecha.

- Estudios donde su título, resumen y palabras clave tuvieran al menos una palabra perteneciente a cada dimensión de la cadena de búsqueda (Qué, Dónde, Integra).

Las fuentes de información que se tuvieron en cuenta fueron: ACM, IEEE, ScienceDirect, Google Academico y Springer. Los resultados de la cadena de búsqueda arrojaron 420 estudios de los cuales 40 reportaban alguna relación directa con el objeto de estudio. Aunque la revisión generó un conjunto amplio de estudios, solo se tuvieron a consideración un subconjunto de 22 propuestas que fueron seleccionadas por cubrir la mayor parte del proceso de diseño de interfaces para promover la accesibilidad en plataformas educativas con énfasis en video. Las demás propuestas seleccionadas, aunque fueron estudiadas y analizadas, no presentan lineamientos de accesibilidad que guíen el diseño de interfaces en plataformas educativas con énfasis en video.

El proceso de revisión sistemática aunque se basa principalmente en los procesos de Kitchenham, aplica el método de caracterización propuesto por González, Moreno, and Martínez (2015) y por Giraldo, España, and Pastor (2014) el cual pretende analizar cada propuesta desde diferentes perspectivas o clasificadores taxonómicos, con el fin de identificar sus fortalezas y brechas en un contexto de actuación. De esta manera, una vez se seleccionaron las propuestas más representativas en relación con el objeto de estudio, se definieron los clasificadores o criterios a partir de las cuales se realizará el análisis de dichas propuestas. La Tabla 1 muestra las preguntas que guiaron la clasificación.

Cada propuesta fue clasificada de acuerdo con los criterios definidos, dando respuesta a las preguntas de investigación planteadas. Como se observa en la Tabla 1, cada pregunta de investigación tiene una correlación con los criterios de clasificación aplicados a las propuestas evaluadas. A partir de la clasificación y análisis se pudo evidenciar las fortalezas de dichas propuestas y posibles brechas encontradas. Una vez efectuada la clasificación se realizó un análisis de cada pregunta de investigación. Finalmente, como resultado del análisis se identifica un interés con el cual constituye el foco de investigación para trabajos futuros. 
Tabla 1

Criterio de caracterización de propuestas evaluadas

Pregunta 1: ¿Qué artefactos evidencia la literatura para promover la accesibilidad en los diversos sistemas interactivos y qué tipo de enfoque utilizan estos estudios?

\begin{tabular}{|c|c|c|}
\hline \multirow{8}{*}{$\begin{array}{l}\text { ¿El estudio ofrece un artefacto para promover la accesibilidad en sistemas } \\
\text { interactivos? }\end{array}$} & Sí & \\
\hline & No & \\
\hline & \multirow{6}{*}{ Tipo de artefacto } & Norma \\
\hline & & Estándar \\
\hline & & Guía \\
\hline & & Metodología \\
\hline & & Modelo \\
\hline & & Otros \\
\hline \multirow{6}{*}{ ¿El estudio se enfoca en una discapacidad específica? } & Sí & \\
\hline & No & \\
\hline & \multirow{4}{*}{ Tipo de discapacidad } & Visual \\
\hline & & Auditiva \\
\hline & & Motriz \\
\hline & & Intelectual \\
\hline \multirow{7}{*}{ ¿El estudio se enfoca en una plataforma específica? } & Sí & \\
\hline & No & \\
\hline & \multirow{5}{*}{ Tipo de plataformas } & Educativas \\
\hline & & Gubernamentales \\
\hline & & Estándar \\
\hline & & Enfoque en video \\
\hline & & Web en general \\
\hline \multirow{5}{*}{ ¿El estudio tiene un enfoque de accesibilidad? } & Sí & \\
\hline & No & \\
\hline & \multirow{3}{*}{ Tipo de enfoque } & Edición de video \\
\hline & & Contenidos Multimedia \\
\hline & & Información \\
\hline \multicolumn{3}{|c|}{ Pregunta 2. ¿Qué nivel de formalismo y tipo de validación tienen las propuestas evaluadas? } \\
\hline \multirow{4}{*}{ ¿El estudio ha sido validado? } & Sí & \\
\hline & No & \\
\hline & \multirow{2}{*}{ Tipo de validación } & Empírica \\
\hline & & No empírica \\
\hline \multirow{5}{*}{ ¿El estudio tiene algún nivel de formalismo? } & Sí & \\
\hline & No & \\
\hline & \multirow{3}{*}{ Nivel de formalismo } & Alto $^{1}$ \\
\hline & & Medio $^{2}$ \\
\hline & & Bajo $^{3}$ \\
\hline \multirow{2}{*}{ ¿El estudio implementa una metodología? } & Sí & \\
\hline & No & \\
\hline \multicolumn{3}{|c|}{ Pregunta 3. ¿Qué herramientas que promuevan la accesibilidad son resultados de las propuestas evaluadas? } \\
\hline \multirow{6}{*}{$\begin{array}{l}\text { ¿El estudio implementa herramientas que ayudan a promover la } \\
\text { accesibilidad? }\end{array}$} & Sí & \\
\hline & No & \\
\hline & \multirow{4}{*}{ Tipo de herramienta } & Lectura de pantalla \\
\hline & & Lenguaje de señas \\
\hline & & Atajos desde teclado \\
\hline & & Otros \\
\hline
\end{tabular}

\section{Fuente: Elaboración propia}

1.Alto: Estudios en los cuales se incluyen un proceso técnico, plantillas, justificación , métricas.

${ }^{2}$ Medio: Estudios en los cuales incluye una descripción técnica.

${ }^{3}$ Bajo: Estudios en los que solo presenta una descripción a modo de checklist o heuristicas. 


\section{Desarrollo de la revisión sistémica}

En esta sección se presentan los diferentes estudios seleccionados y su principal aporte, los cuales permiten obtener las primeras contribuciones a las preguntas de investigación previamente planteadas. Posteriormente, se expone la clasificación de las propuestas en cada uno de los criterios de la Tabla 1.

\subsection{Presentación de propuestas seleccionadas}

El trabajo de González, Moreno, and Martínez (2015) presenta un enfoque a los requisitos de accesibilidad siguiendo estándares pre-establecidos, una conceptualización de los requisitos para la integración en el proceso de desarrollo y una verificación de la integración de estos requisitos que promueven el diseño de reproductores multimedia accesibles.

Por otro lado, el trabajo de García (2014) discute sobre la incorporación del video en la educación y formas de volverlo accesible para personas con discapacidad auditiva, haciendo especial énfasis en una buena calidad de subtítulos y la clasificacion de dichos subtítulos.

En este sentido, el estudio de Monedero, Robles, and Desenne (2015) presenta el diseño y creación de herramientas de anotaciones multimedia para mejorar la interactividad de los usuarios con los contenidos. Igualmente, el estudio muestra la evaluación de dos herramientas: Collaborative Annotation Tool (CaTool) y Open Video Annotation (OVA), esta última desarrollada por el proyecto e integrada en el MOOC de EdX enfocados en anotaciones de video para usuarios con discapacidad.

El estudio de Andrade (2012) presenta un resumen sobre la teoría de la carga cognitiva y la teoría cognitiva del aprendizaje multimedia, la forma de abordar este enfoque en las plataformas educativas, la importancia de la duración del video y la manera en la que el estudiante procesa la información.

Del mismo modo, el artículo de Pérez, Lucía. Herranz, M. Abadín, D. and Delgado Santos, C (2016) presenta cómo se debe abordar la accesibilidad cognitiva en entornos multimedia presentando guías para la implementación de estas enfocadas en la teoría de la carga cognitiva.

En el trabajo de Andrade and Chacón (2018) se presenta el concepto de aulas invertidas y la educación basada en la taxonimia de Bloom y el cono de expereiencia de Edgar Dale, definiendo las aulas presenciales como un apredizaje pasivo y las aulas virtuales con un aprendizaje activo.

Por su parte, el trabajo de De la Fuente, Hernández and Pra Martos (2017) muestra la importancia del video en la educación y el buen uso de este, al tiempo que plantean el video como una herramienta de educación adicional a las clases presenciales. Por esta razón, el trabajo de Ramírez Ochoa (2016) destaca la utilidad del video como herramienta docente para la administración de contenidos, constitución de comunidades de aprendizaje y formación de habilidades discentes para la búsqueda, selección, almacenamiento y evaluación de representaciones audiovisuales de autoría propia o extraña.

En el estudio de Hernández (2013) se determina la usabilidad de un software educativo mediante la práctica docente y el nivel de enseñanza y a raíz de esto se propone una herramienta de apoyo en el proceso de enseñanza, en el cual se evalúa el proceso del docente como enseñanza virtual, al medir el aprendizaje del estudiante mediante la dinámica (quiz, talleres, actvidades, foros) de las aulas virtuales.

En el artículo de Díaz et al. (2017) se presentan herramientas tanto para la evaluación de un portal web, como también aplicaciones de ayuda para las personas con discapacidad visual. Adicionalmente, se exponen los errores más típicos presentes en los portales web. 
El estudio de Gómez, C. and Cobos Pérez R (2013) argumentan que la reutilización de recursos existentes combinados con métodos y prácticas colaborativas, permiten la manipulación y composición de objetos de aprendizaje multimedia-interactivos.

En el estudio de Piedra et al. (2013) describen el marco de trabajo en el que se deben ejecutar procesos de creación, produción y reutilización de materialesn multimedia educativos accesibles, así como el proceso de implementación de estos materiales en entornos educativos accesibles. Igualmente, se describe la estructura de estos procesos en términos de actividades y tareas, y se plantean un conjunto de técnicas y métodos para aplicar, con el fin de asegurar el cumplimiento de métricas y criterios de calidad.

Los autores Gil et al. (2016) presentan el estudio de accesibilidad en MOOC tanto de las herramientas tecnológicas integradas en los cursos de fácil comprensión, como de los aspectos relacionados con inclusión y posibilidades para personas en situación de desventaja social, limitaciones de conectividad o pocas habilidades en las TIC y de bajo o nulo conocimiento en otros idiomas.

De otro modo, el estudio Sánchez and Mora (2015) propone una adaptación de la metodología de evaluación de la accesibilidad web WCAG-EM para su utilización en contenidos educativos para aprendizaje en línea.

En Peñafiel and Mora (2014) se presenta un estudio preliminar respecto de la legislación sobre la accesibilidad web en países como España, Uruguay, Colombia, Chile, Brasil y Ecuador, en el cual se presenta la información debidamente sistematizada en función de la madurez alcanzada en este ámbito de la accesibilidad por los países participantes.

Por otra parte, el estudio de Pagnoni and Mariño (2019) hace referencia al cumplimiento de los estándares establecidos según el World Wide Web Consortium (W3C) respecto de la Accesibilidad Web (AW), aplicando dos validadores a una página web perteneciente a un portal educativo estatal.

Del mismo modo en Baños García et al., (2018) se presenta una guía para el uso de material multimedia en la pedagogía teniendo en cuenta los principios de Mayer ${ }^{1}$ para lograr una mayor inclusión de los estudiantes.

En este orden de ideas, el trabajo de Oliveira and Silva (2019) define un conjunto de pautas de accesibilidad del concepto y práctica de la descripción de audio, para guiar el desarrollo de diferentes recursos educativos de uso común, en cursos de aprendizaje a distancia.

En el estudio de González, Moreno, and Martínez (2015) se presenta un checklist para evaluar el contenido multimedia en sitios web desde cuatro macro categorías diferentes: forma, contenido, lenguaje y actividad.

El documento de Distrital and Tolerancia (2019) presenta los lineamientos de accesibilidad edificados bajo tres puntos específicos, en los cuales se presentan las mayores fallas tales como: 1) bajo nivel de incidencia de las instancias de participación ciudadana, 2) debilidad en la entrega de información en los procesos de rendición de cuentas, 3) debilidad en la articulación de sistemas de información y acceso a la información pública.

\subsection{Clasificación y análisis de propuesta}

La clasificación de las propuestas se hizo por medio de los criterios especificados en la Tabla 1, los cuales dan respuesta a las preguntas de investigación. A continuación, se presenta la clasificación de las propuestas

\footnotetext{
${ }^{1}$ Fueron diseñados con el fin de trabajar en entornos digitales como: Principio de Coherencia, Señalización, Redundancia, Contigüidad Espacial, Contigüidad Temporal, Segmentación, Pre-formacion, Modalidad, Multimedia, Personalización, Voz e Imagen.
} 
evaluadas de acuerdo con cada criterio (Tablas 2 a la 9) y el análisis de fortalezas y brechas encontradas a partir de la clasificación.

\section{P1: ¿Qué artefactos evidencia la literatura para promover la accesibilidad en los diversos sistemas interactivos y qué tipos de enfoque utilizan estos estudios?}

Tal como se expuso en la sección de metodología, para la pregunta de investigación en cuestión se definieron los siguientes criterios de clasificación a) Artefactos b) Enfoque de discapacidad c) Plataforma d) Enfoque de accesibilidad. A continuación, se expone el análisis para cada uno de los criterios

a) ¿El estudio ofrece un artefacto para promover la accesibilidad en sistemas interactivos? Los artefactos hacen referencia a los resultados que ofrecen los estudios evaluados, los cuales podían ser: normas de accesibilidad, estándares de accesibilidad, guías de accesibilidad, metodologías o modelos de accesibilidad, entre otros.

En la tabla 2 se presenta la clasificación de las propuestas de acuerdo con el criterio previamente definido. Como se observa en la tabla 2, la mayoría de los estudios (19 en total) ofrecen artefactos para promover la accesibilidad en sistemas interactivos, siendo las guías el artefacto más común para la implementación de contenidos accesibles. Entre los artefactos clasificados como otros se encuentran: principios, acuerdos, checklist y prácticas.

La mayoría de los artefactos estudiados están diseñados para promover la accesibilidad en páginas web en general, es decir, no se enfocan en un tipo de contexto específico (educación, gobierno, turismo, etc.). Este tipo de guías son necesarias pues ofrecen una visión general de como promover la accesibilidad en contextos, por ejemplo, como el de las plataformas educativas basadas en video. Sin embargo, las guías para contextos específicos son igualmente necesarias debido a que cada contexto tiene sus particularidades. Igualmente se resalta que no se evidencia en los diferentes tipos de lineamientos de accesibilidad una descripción respecto de los beneficios de la implementación de estos.

Tabla 2

Criterio de clasificación: artefacto

\begin{tabular}{|c|c|c|c|}
\hline \multirow{8}{*}{$\begin{array}{l}\text { ¿El estudio ofrece } \\
\text { un artefacto para } \\
\text { promover la } \\
\text { accesibilidad en } \\
\text { sistemas } \\
\text { interactivos? }\end{array}$} & Sí & \multicolumn{2}{|c|}{$\begin{array}{l}\text { González, Moreno, and Martínez (2015) Peñafiel and Luján-Mora (2014) Hernández (2013)García } \\
\text { (2014)Pagnoni and Mariño (2019)Gómez and Pérez (2013)Monedero, Robles and Desenne } \\
\text { (2015)Baños García et al (2018)Pérez-Castilla Álvarez et al. (2016)Diaz et al.( 2017)Oliveira and Silva } \\
\text { (2019)Moreno et al. (2017)Piedra et al. (2013)Distrital and Tolerancia (2019)Gómez and Cobos } \\
\text { (2018)Gil et al. (2016)Sanchez and Mora (2015)De la Fuente, Hernández and Pra Martos (2017) }\end{array}$} \\
\hline & No & \multicolumn{2}{|c|}{ (Andrade-Lotero 2012) } \\
\hline & \multirow{6}{*}{$\begin{array}{l}\text { Tipo de } \\
\text { artefacto }\end{array}$} & Norma & \\
\hline & & Estándar & Peñafiel and Luján-Mora (2014) \\
\hline & & Guía & $\begin{array}{l}\text { Pérez-Castilla Álvarez et al. (2016) Oliveira and Silva } \\
\text { (2019) Gómez and Cobos (2018) Gil et al. (2016) De la } \\
\text { Fuente, Hernández and Pra Martos (2017) }\end{array}$ \\
\hline & & Metodología & $\begin{array}{l}\text { Gómez and Pérez (2013)Monedero, Robles, and } \\
\text { Desenne (2015) Sanchez and Mora (2015) }\end{array}$ \\
\hline & & Modelo & González, Moreno, and Martínez (2015) García (2014) \\
\hline & & Otros & $\begin{array}{l}\text { Hernández (2013) Pagnoni and Mariño (2019) García, } \\
\text { Lezcano, and Muñoz (2018) Diaz et al. (2017) Moreno } \\
\text { et al. (2017) Distrital and Tolerancia (2019) }\end{array}$ \\
\hline
\end{tabular}

Fuente: Elaboración propia

b) ¿El estudio se enfoca en una discapacidad específica? El enfoque de discapacidad agrupa las categorías visuales, auditivas, motrices e intelectuales. Este criterio de clasificación fue primordial debido que en este se logra identificar la discapacidad o disparidades foco de investigación de los estudios evaluados. 
En la Tabla 3 se observa que la mayoría de las propuestas evaluadas no se enfocan en una discapacidad específica.

De otro modo, las propuestas que se enfocan en una discapacidad están enmarcadas, en su mayoría, en la discapacidad visual y auditiva. De esta amanera, se observa que son pocos los estudios enfocados en la discapacidad motriz e intelectual. Adicionalmente, las propuestas que no se enfocan en una discapacidad se centran en agrupar sus aportes según los principios de accesibilidad (perceptible, operable, compresible y robusto). De esta manera, es importante tener lineamientos descritos a partir de ambos enfoques, así se brindan mayores posibilidades de aplicación y se promueve minimizar la brecha de exclusión para personas con discapacidad.

Tabla 3

Criterio de clasificación: enfoque de discapacidad

\begin{tabular}{|c|c|c|}
\hline \multirow{6}{*}{$\begin{array}{l}\text { ¿El estudio se } \\
\text { enfoca en una } \\
\text { discapacidad } \\
\text { específica? }\end{array}$} & Sí & $\begin{array}{l}\text { (García 2014)(Andrade-Lotero 2012) (Baños García et al., 2018) (Diaz et al. 2017)(Oliveira and } \\
\text { Silva 2019(Distrital and Tolerancia 2019)(Ministerio de Educación Nacional 2018)(Gil et al. 2016) }\end{array}$ \\
\hline & No & $\begin{array}{l}\text { (González, Moreno, and Martínez 2015)(Peñafiel and Luján-Mora 2014)(Hernández } \\
\text { 2013)(Pagnoni and Mariño 2019)(Gómez and Cobos 2013)(Monedero-Moya, Cebrián-Robles, and } \\
\text { Desenne 2015)(Pérez-Castilla Álvarez et al. 2016)(Piedra et al. 2013)(Sanchez Gordon and Luján } \\
\text { Mora 2015) }\end{array}$ \\
\hline & \multirow{4}{*}{$\begin{array}{c}\text { Tipo de } \\
\text { discapacidad }\end{array}$} & \begin{tabular}{l|l} 
Visual & $\begin{array}{l}\text { (Baños García et al., 2018) (Diaz et al. 2017)(Oliveira } \\
\text { and Silva 2019) (Distrital and Tolerancia 2019) } \\
\text { Gómez and Cobos (2013) (Gil et al. 2016) }\end{array}$
\end{tabular} \\
\hline & & $\begin{array}{l}\text { (García 2014) (Baños García et al., 2018) (Distrital } \\
\text { and Tolerancia 2019) Gómez and Cobos (2013) (Gil } \\
\text { et al. 2016a) }\end{array}$ \\
\hline & & (Gil et al. 2016) \\
\hline & & (Andrade-Lotero 2012) (Gil et al. 2016) \\
\hline
\end{tabular}

Fuente: Elaboración propia

c) ¿El estudio se enfoca en una plataforma específica? La mayoría de los estudios se enfocan en una plataforma específica y 16 de estos se centran en plataformas educativas.

El principal aporte de las propuestas enfocadas en plataformas educativas es aplicar los principios de accesibilidad y niveles de conformidad propuestos por los estandares y recomendaciones del W3C ( $A, A A, A A A)$. Aunque aplicar dichos principios y pautas en una plataforma promueve la accesibilidad, es necesario contar con guías o lineamientos específicos para cada tipo de sistema interactivo, de tal manera que especifique lo necesario para cada de una de las diferentes discapacidades. 
Tabla 4

Criterio de clasificación: plataforma

\begin{tabular}{|c|c|c|c|}
\hline \multirow{7}{*}{$\begin{array}{l}\text { ¿El estudio se } \\
\text { enfoca en una } \\
\text { plataforma } \\
\text { específica? }\end{array}$} & Sí & \multicolumn{2}{|c|}{$\begin{array}{l}\text { (González, Moreno, and Martínez 2015)(Peñafiel and Mora 2014)(García 2014)(Andrade- } \\
\text { Lotero 2012)(Pagnoni and Mariño 2019)(Gómez and Pérez 2013)(Monedero, Robles and } \\
\text { Desenne 2015)(García, Lezcano and Muñoz 2018)(Pérez-Castilla Álvarez et al. 2016)(Diaz et al. } \\
\text { 2017)(Oliveira and Silva 2019)(Moreno et al. 2017)(Piedra et al. 2013)(Distrital and Tolerancia } \\
\text { 2019)(Ministerio de Educación Nacional 2018)(Gil et al. 2016)(Sanchez and Mora 2015) }\end{array}$} \\
\hline & No & \multicolumn{2}{|c|}{ (Hernández 2013) } \\
\hline & \multirow{5}{*}{$\begin{array}{l}\text { Tipo de } \\
\text { plataformas }\end{array}$} & Educativas & $\begin{array}{l}\text { (García 2014) (Andrade-Lotero 2012) (Pagnoni and } \\
\text { Mariño 2019) (Monedero, Robles and Desenne } \\
\text { 2015) (Baños García et al., 2018) (Diaz et al. 2017) } \\
\text { (Oliveira and Silva 2019) (Piedra et al. 2013a) } \\
\text { (Distrital and Tolerancia 2019) (Ministerio de } \\
\text { Educación Nacional 2018) (Gil et al. 2016)(Sanchez } \\
\text { and Mora 2015) }\end{array}$ \\
\hline & & Gubernamentales & \\
\hline & & estándares & \\
\hline & & Enfoque en video & $\begin{array}{l}\text { (González, Moreno, and Martínez 2015) (Gómez } \\
\text { and Cobos 2013) }\end{array}$ \\
\hline & & Web en general & $\begin{array}{l}\text { (Peñafiel and Mora 2014) (Pérez-Castilla Álvarez et } \\
\text { al. 2016)(Moreno et al. 2017) (Gil et al. 2016) }\end{array}$ \\
\hline
\end{tabular}

Fuente: Elaboración propia

d) ¿El estudio tiene un enfoque de accesibilidad? Este criterio de clasificación está orientado a determinar si las propuestas evaluadas se enfocan en promover la accesibilidad en algún elemento de la interfaz de usuario. En las propuestas que tienen algún tipo de enfoque se evidenció que promueven la accesibilidad en la edición de video, en los contenidos multimedia y en la información textual.

\section{Tabla 5}

Criterio de clasificación: enfoque de accesibilidad

\begin{tabular}{|c|c|c|c|}
\hline \multirow{5}{*}{$\begin{array}{l}\text { ¿El estudio tiene un } \\
\text { enfoque de } \\
\text { accesibilidad? }\end{array}$} & Sí & \multicolumn{2}{|c|}{$\begin{array}{l}\text { (González, Moreno, and Martínez 2015)(Peñafiel and Mora 2014)(Hernández 2013)(García } \\
\text { 2014)(Pagnoni and Mariño 2019) (Gómez and Pérez 2013) (Monedero, Robles and } \\
\text { Desenne 2015) (García, Barbero, and Muñoz 2018)(Pérez-Castilla et al. 2016) (Diaz et al. } \\
\text { 2017) (Oliveira and Silva 2019) (Moreno et al. 2017)(Piedra et al. 2013) (Distrital and } \\
\text { Tolerancia 2019) (Ministerio de Educación Nacional 2018) (Gil et al. 2016) (Sánchez and } \\
\text { Mora 2015) }\end{array}$} \\
\hline & No & \multicolumn{2}{|l|}{ (Andrade-Lotero 2012) } \\
\hline & \multirow{3}{*}{$\begin{array}{l}\text { Tipo de } \\
\text { enfoque }\end{array}$} & Edición de video & (García 2014) (Monedero, Robles and Desenne 2015) \\
\hline & & Contenidos Multimedia & $\begin{array}{l}\text { (González, Moreno, and Martínez 2015) (Gómez and Pérez } \\
\text { 2013) (García, Barbero, and Muñoz 2018) (Moreno et al. } \\
\text { 2017)(Piedra et al. 2013) (Ministerio de Educación Nacional } \\
\text { 2018) (Gil et al. 2016) (Sánchez and Mora 2015) }\end{array}$ \\
\hline & & Información & $\begin{array}{l}\text { (Peñafiel and Mora 2014) (Hernández 2013) (Pagnoni and } \\
\text { Mariño 2019) (Pérez-Castilla et al. 2016) (Diaz et al. 2017) } \\
\text { (Oliveira and Silva 2019) (Distrital and Tolerancia 2019) }\end{array}$ \\
\hline
\end{tabular}

Fuente: Elaboración propia

Como se observa en la Tabla 6, la mayoría de las propuestas se enfocan en promover la accesibilidad en los contenidos multimedia, específicamente en el diseño, edición y presentación de imágenes estáticas, imágenes en movimiento o gifs, audios y videos. De dichas propuestas se destaca una guía para la realización de subtítulos y uso de transcripciones (Ramírez Ochoa, 2016). No obstante, se observa la brecha de exclusión para personas con poca movilidad debido a que las propuestas orientadas a contenidos multimedia no contemplan el acceso 
por medio de patrones de control de teclado o el uso de herramientas externas al hardware convencional. Igualmente, se observa carencia de guías orientadas a promover la accesibilidad en el proceso interactivo durante la ejecución de una actividad o tarea.

\section{P2: ¿Qué nivel de formalismo y tipo de validación tienen las propuestas evaluadas?}

Para la pregunta de investigación en cuestión, los criterios de clasificación aplicados fueron: a) validación b) formalismo c) metodología.

a) ¿El estudio ha sido validado? Las propuestas evaluadas que tuvieron algún tipo de validación se pudieron clasificar en dos tipos:

i. Empírica: aquellos estudios que realizaban algún tipo de experimentación.

ii. No empírica: aquellos estudios que no tuvieron ningún tipo de experimentación.

Este criterio fue primordial ya que se logra identificar la veracidad de la experimentación de los diferentes estudios realizados.

Como se evidencia en la Tabla 6, 17 de los estudios tienen una validación empírica, en los cuales se destaca el realizar pruebas usando enfoques de evaluacion como evaluaciones heurísticas y evaluaciones de accesibilidad y desempeño con expertos. No obstante, no se logra evidenciar pruebas de concepto o de participación de usuarios finales en dichas pruebas.

\section{Tabla 6}

Criterio de clasificación: validación

\begin{tabular}{|c|c|c|c|}
\hline \multirow{4}{*}{$\begin{array}{l}\text { ¿El estudio ha sido } \\
\text { validado? }\end{array}$} & Sí & \multicolumn{2}{|c|}{$\begin{array}{l}\text { (González, Moreno, and Martínez 2015)(Hernández 2013)(García 2014)(Andrade-Lotero } \\
\text { 2012)(Pagnoni and Mariño 2019)(Gómez and Pérez 2013)(Monedero, Robles and Desenne } \\
\text { 2015)(Peñafiel and Mora 2014)(García, Barbero, and Muñoz 2018)(Pérez-Castilla et al. } \\
\text { 2016)(Diaz et al. 2017)(Oliveira and Silva 2019)(Piedra et al. 2013)(Distrital and Tolerancia } \\
\text { 2019)(Ministerio de Educación Nacional 2018)(Gil et al. 2016)(Sánchez and Mora 2015) }\end{array}$} \\
\hline & No & \multicolumn{2}{|c|}{ (Hernández 2013)(Moreno et al. 2017) } \\
\hline & \multirow[t]{2}{*}{$\begin{array}{l}\text { Tipo de } \\
\text { validación }\end{array}$} & Empírica & $\begin{array}{l}\text { (Andrade-Lotero 2012) (Pagnoni and Mariño 2019) (Gómez and } \\
\text { Pérez 2013) (Monedero, Robles and Desenne 2015) (González, } \\
\text { Moreno, and Martínez 2015) (García, Barbero, and Muñoz 2018) } \\
\text { (Pérez-Castilla et al. 2016) (Diaz et al. 2017) (Oliveira and Silva } \\
\text { 2019) (Piedra et al. 2013) (Distrital and Tolerancia 2019) } \\
\text { (Ministerio de Educación Nacional 2018) (Gil et al. 2016) (Sánchez } \\
\text { and Mora 2015) }\end{array}$ \\
\hline & & No empírica & (García 2014) (García 2014) \\
\hline
\end{tabular}

Fuente: Elaboración propia

b) ¿El estudio tiene algún nivel de formalismo? Frente a este criterio se especificaron tres categorías, en las cuales se identificó el nivel de formalismo de los lineamientos de accesibilidad asociados a las propuestas evaluadas; así las categorías, asociadas son:

i. Alto: aquellos estudios que incluyen en los lineamientos de accesibilidad un proceso, técnicas, justificación y métricas de aplicación.

ii. Medio: aquellos estudios que incluyen en los lineamientos de accesibilidad descripción de técnicas o métodos.

iii. Bajo: Aquellos estudios que describen los lineamientos de accesibilidad a modo de checklist o heurísticas. 
Tabla 7

Criterio de clasificación: nivel de formalismo

\begin{tabular}{|c|c|c|c|}
\hline \multirow{5}{*}{$\begin{array}{l}\text { ¿El estudio tiene } \\
\text { algún nivel de } \\
\text { formalismo? }\end{array}$} & Sí & \multicolumn{2}{|c|}{$\begin{array}{l}\text { (González, Moreno, and Martínez 2015) (Hernández 2013) (García 2014) (Andrade- } \\
\text { Lotero 2012) (Andrade-Lotero 2012) (Gómez and Pérez 2013) (Monedero, Robles and } \\
\text { Desenne 2015) (Pérez-Castilla et al. 2016) (Oliveira and Silva 2019)(Moreno et al. } \\
\text { 2017)(Piedra et al. 2013) (Distrital and Tolerancia 2019) (Ministerio de Educación } \\
\text { Nacional 2018) (Gil et al. 2016) (Sánchez and Mora 2015) }\end{array}$} \\
\hline & No & \multicolumn{2}{|c|}{ (Peñafiel and Mora 2014) (Diaz et al. 2017) } \\
\hline & \multirow{3}{*}{$\begin{array}{l}\text { Nivel de } \\
\text { formalismo }\end{array}$} & Alto & (Distrital and Tolerancia 2019) \\
\hline & & Medio & $\begin{array}{l}\text { (González, Moreno, and Martínez 2015) (Gómez and Pérez } \\
\text { 2013) (Monedero, Robles and Desenne 2015) (Oliveira and } \\
\text { Silva 2019)(Piedra et al. 2013) (Ministerio de Educación } \\
\text { Nacional 2018) (Gil et al. 2016) }\end{array}$ \\
\hline & & Bajo & $\begin{array}{l}\text { (Hernández 2013)(García 2014) (Andrade-Lotero 2012) } \\
\text { (Andrade-Lotero 2012) (Pérez-Castilla et al. 2016)(Moreno et } \\
\text { al. 2017) (Sánchez and Mora 2015) }\end{array}$ \\
\hline
\end{tabular}

Fuente: Elaboración propia

Como se observa en la tabla 7, solo hay una propuesta que tiene un alto nivel de formalismo. De esta manera, se evidencian falencias en la mayoría de propuestas evaluadas en relación a este criterio. Esto se debe a que mientras mayor sea el nivel de formalismo del lineamiento menor es la subjetividad de la persona que lo aplica. De igual manera, un alto nivel de formalidad permite que las personas que aplican los lineamientos lo puedan hacer de manera autónoma.

c) ¿El estudio implementa una metodología? Con este criterio lo que se esperaba, más que una clasificación, era identificar y conocer metodologías aplicadas a la accesibilidad de portales educativos con énfasis en video. En la tabla 8, se pueden observar las diferentes metodologías identificadas.

Tabla 8

Criterio de clasificación: implementación de metodologías

\begin{tabular}{|c|c|c|}
\hline \multirow{3}{*}{$\begin{array}{l}\text { ¿El estudio } \\
\text { implementa una } \\
\text { metodología? }\end{array}$} & Sí & $\begin{array}{l}\text { (González, Moreno, and Martínez 2015) (García 2014) (Andrade-Lotero 2012)(Gómez and } \\
\text { Pérez 2013) (Monedero, Robles and Desenne 2015)(García, Barbero, and Muñoz 2018) } \\
\text { (García, Barbero, and Muñoz 2018) (Pérez-Castilla et al. 2016) (Diaz et al. 2017)(Oliveira and } \\
\text { Silva 2019)(Moreno et al. 2017) (Piedra et al. 2013) (Distrital and Tolerancia } \\
\text { 2019)(Ministerio de Educación Nacional 2018)(Gil et al. 2016) (Sánchez and Mora 2015) }\end{array}$ \\
\hline & No & (Peñafiel and Mora 2014) (Hernández 2013) \\
\hline & $\begin{array}{l}\text { ¿Qué tipo de } \\
\text { metodología? }\end{array}$ & $\begin{array}{l}\text { (González, Moreno, and Martínez 2015) UAAG } \\
\text { (García 2014) Norma UNE 153010:2012 } \\
\text { (Andrade-Lotero 2012) Teoria de la Carga cognitiva, Teoría del aprendizaje multimedia } \\
\text { (Gómez and Pérez 2013) Social Media Learning (SMLerning) } \\
\text { (Monedero, Robles and Desenne 2015) Herramamientas CaTool } \\
\text { (García, Barbero, and Muñoz 2018) Metodologia ApS } \\
\text { (Pérez-Castilla et al. 2016) Accesibilidad Cognitiva } \\
\text { (Oliveira and Silva 2019) Ambiente Virtual de Ensino e Aprendizaje } \\
\text { (Moreno et al. 2017) (Gil et al. 2016) WCAG } 2.0 \\
\text { (Piedra et al. 2013) Proceso de concepción de diseño } \\
\text { (Distrital and Tolerancia 2019)(Ministerio de Educación Nacional 2018) NTC } 5458 \text { Norma } \\
\text { Técnica Colombiana de accesibilidad } \\
\text { (Sánchez and Mora 2015) WCAG-EM }\end{array}$ \\
\hline
\end{tabular}

Fuente: Elaboración propia 


\section{P3: ¿Qué herramientas que promueven la accesibilidad son resultados de las propuestas evaluadas?}

Frente a la pregunta de investigación anteriormente plateada el criterio de clasificación asociado es: a) herramientas de accesibilidad.

a) ¿El estudio implementa herramientas que ayudan a promover la accesibilidad? Este criterio busca identificar las diferentes herramientas con las cuales se puede incrementar la accesibilidad de plataformas educativas basadas en video.

Tabla 9

Criterio de clasificación: herramientas

\begin{tabular}{|c|c|c|c|}
\hline \multirow{6}{*}{$\begin{array}{l}\text { ¿El estudio implementa } \\
\text { herramientas que ayudan a } \\
\text { promover la accesibilidad? }\end{array}$} & Sí & \multicolumn{2}{|c|}{$\begin{array}{l}\text { (González, Moreno, and Martínez 2015) (Hernández 2013) (García 2014) } \\
\text { (Pagnoni and Mariño 2019) (Monedero, Robles and Desenne 2015) (Diaz et } \\
\text { al. 2017) (Oliveira and Silva 2019) (Gil et al. 2016) }\end{array}$} \\
\hline & No & \multicolumn{2}{|c|}{$\begin{array}{l}\text { (Peñafiel and Mora 2014) (Andrade-Lotero 2012) (Gómez and Pérez 2013) } \\
\text { (García, Barbero, and Muñoz 2018) (Pérez-Castilla et al. 2016) (Moreno et } \\
\text { al. 2017) (Piedra et al. 2013) (Distrital and Tolerancia 2019) (Ministerio de } \\
\text { Educación Nacional 2018) (Sánchez and Mora 2015) }\end{array}$} \\
\hline & \multirow{4}{*}{$\begin{array}{c}\text { Tipo de } \\
\text { herramienta }\end{array}$} & Lectura de pantalla & (Gil et al. 2016) \\
\hline & & Lenguaje de señas & \\
\hline & & Atajos desde teclado & (Gil et al. 2016) \\
\hline & & Otros & $\begin{array}{l}\text { (González, Moreno, and Martínez 2015) } \\
\text { (Hernández 2013) (García 2014) (Pagnoni and } \\
\text { Mariño 2019) (Monedero, Robles and Desenne } \\
\text { 2015) (Diaz et al. 2017) (Oliveira and Silva 2019) }\end{array}$ \\
\hline
\end{tabular}

Fuente: Elaboración propia

Como se observa en la Tabla 9, 8 estudios de las propuestas evaluadas cuentan con alguna herramienta para promover la accesibilidad, entre las que se destacan: lectores de pantalla, uso de simuladores de discapacidad para lograr entender las posibles dificultades que presentan este tipo de usuarios, validadores o test de accesibilidad.

\section{Discusión}

De acuerdo con el estudio realizado se observó que se evidencian propuestas relacionadas con lineamientos para promover la accesibilidad en plataformas educativas. La mayoría de estas propuestas cubren el desarrollo de contenidos educativos accesibles y solo unas pocas cubren el desarrollo de contenidos educativos basados en el video. Igualmente, se evidenció que la mayoría de los lineamientos de accesibilidad son generales, es decir, no se enfocan en una discapacidad o plataforma específica. Aquellos que tienen algún tipo de enfoque están más orientados hacia la discapacidad visual y auditiva. Lo anterior supone una carencia en relación a lineamientos de accesibilidad enfocados a discapacidades como la motriz e intelectual o enfocados a plataformas de dominios específicos como la educación. Del mismo modo, se observan carencias en relación al nivel de formalismo de los lineamientos, lo cual genera subjetividad por parte de las personas que aplican dichos lineamientos debido a que no existen procesos, técnicas, métricas o una descripción detallada para su implementación. Así mismo, el bajo nivel de formalismo repercute en la implementación de dichos lineamientos debido a que las personas no lo puedan aplicar de forma autónoma.

De otro modo, se evidencia que los lineamientos de accesibilidad deberían estar clasificados de acuerdo con diferentes taxonomías, cada una de ellas orientada a identificar el contexto de aplicación de acuerdo a una necesidad particular. Una descripción deseada de un lineamiento de accesibilidad debería incluir este aspecto, es decir, una clasificación del lineamiento de acuerdo con, por ejemplo, la discapacidad, nivel de conformidad, tipo de plataforma, etc. En este sentido, se abre una oportunidad para la definición de lineamientos para el 
contexto particular de las plataformas educativas basadas en video, los cuales tengan en cuenta los diferentes criterios definidos en el presente artículo.

\section{Conclusiones}

Hoy en día, la educación virtual, a distancia e incluso la presencial, ha sido impactada por las diferentes herramientas y recursos didácticos mediados por las TIC. Uno de los recursos más utilizado son los contenidos educativos en formato de video y herramientas que permiten su reproducción, exploración y análisis como marcadores, transcripciones, notas, índices, etc. De acuerdo a lo anterior, los sistemas de gestión de aprendizaje (LMS - Learning Management System) deben propender a que estas herramientas y recursos sean de fácil acceso para todos los usuarios, incluidos aquellos que tienen discapacidad, con el fin de disminuir la brecha de exclusión. Teniendo en cuenta este contexto, se realizó una revisión sistemática de la literatura respecto de propuestas enfocadas en lineamientos de accesibilidad para plataformas educativas que tuvieran énfasis en contenidos de video. Dichas propuestas fueron clasificadas de acuerdo con diferentes aspectos. La clasificación permitió identificar las fortalezas y brechas para suplir en trabajos futuros.

Dentro de las fortalezas identificadas en el estudio, se evidenció literatura extensa en relación con lineamientos de accesibilidad de aplicación general, es decir sin enfocarse en una discapacidad o plataforma específica. Igualmente, se evidencian propuestas para la discapacidad visual y auditiva. Dentro de las brechas identificadas están: escasas propuestas enfocadas hacia la discapacidad motriz o intelectual, bajo formalismo y poca documentación en relación a la implementación y evaluación de cada lineamiento. Posibles trabajos futuros podrían estar enfocados en definir lineamientos de accesibilidad para la discapacidad motriz e intelectual, que sean formales (describe su objetivo, justificación, contexto de uso, proceso, técnicas, métricas) y de aplicación en el contexto educativo.

La revisión realizada permitió determinar un posible aporte en cuanto a la definición de lineamientos de accesibilidad que permitan guiar el proceso de diseño e implementación de plataformas educativas basadas en video, las cuales aprovechen las falencias identificadas en el estudio, en cuanto al formalismo, el tipo de discapacidad considerada y la especificidad de cada lineamiento.

\section{Referencias}

Andrade, E and Chacón, E. 2018. "Implicaciones Teóricas y Procedimentales de La Clase Invertida." PULSO. Revista de Educación 0(41): 251-67.

Andrade Lotero, L. 2012. "Teoría de La Carga Cognitiva, Diseño Multimedia y Aprendizaje: Un Estado Del Arte." Magis 5(10): 75-92.

Baños García, et al. (2018). Materiales multimedia: Diseño desde una pedagogía inclusiva. European Journal of Child Development, Education and Psychopathology, 6(2), 107. https://doi.org/10.30552/ejpad.v6i2.73

Baños García, M. E., Lezcano Barbero, F., \& Casado Muñoz, R. (2018). Materiales multimedia: Diseño desde una pedagogía inclusiva. European Journal of Child Development, Education and Psychopathology, 6(2), 107. https://doi.org/10.30552/ejpad.v6i2.73

Belloch, C. 2012. "Las Tecnologías de La Información y Comunicación En El Aprendizaje." Departamento de Metodos de Investigacion y Diagnostico en Eduacioón. https://www.uv.es/bellochc/pedagogia/EVA1.wiki (August 2, 2020).

Gómez, C. and Cobos Pérez, R. 2013. "Del Vídeo Educativo a Objetos de Aprendizaje Multimedia Interactivos: Un Entorno de Aprendizaje Colaborativo Basado En Redes Sociales." Tendencias pedagógicas (22): 59-72. 
De la Fuente Sánchez, D. Hernández Solís, M and Pra Martos, I 2017. “Vídeo Educativo y Rendimiento Académico En La Enseñanza Superior a Distancia." RIED. Revista Iberoamericana de Educación a Distancia 21(1): 323.

Diaz, F. Schiavoni,A. Amadeo,A. and Ray,D. 2017. "Making a Teaching Material Repository Accessible. An Experience on an Open Source Platform." Proceedings of the 2016 42nd Latin American Computing Conference, CLEI 2016.

Distrital and Tolerancia. 2019. "Lineamiento de Accesibilidad Web Para Sitios Web." Alcaldia Mayor de Bogota.

García, P.J. 2014. "Video in Education: Create Subtitles to Break Accessibility Barriers." International Journal of Edutational Research and Innovation (IJERI) 2: 107-17.

García Pérez, M, and Ortega Sánchez, M. 2010. “Atención a La E-Accesibilidad y Usabilidad Universal En El Diseño Formativo." Pixel-Bit

Gil, A. et al. 2016. "Construction of Management Capacities of MOOC in Higher Education Informe Sobre Accesibilidad Aplicada a MOOC."

Giraldo. F. , España. S , and Pasto, O. 2014. "Analysing the Concept of Quality in Model-Driven Engineering Literature: A Systematic Review." Proceedings - International Conference on Research Challenges in Information Science.

González, M. Moreno, L. , and Martínez, P. 2015. "Approach Design of an Accessible Media Player." Universal Access in the Information Society 14(1): 45-55.

Hernández, A. 2013. "Usabilidad de Un Software Educativo Como Medio Instruccional Para El Proceso de Enseñanza-Aprendizaje de Una Asignatura." Revista Iberoamericana para la Investigación y el Desarrollo Educativo (11). http://www.ride.org.mx/1-11/index.php/RIDESECUNDARIO/article/viewFile/672/658.

Hernandez, R. 2017. "Impacto de Las TIC En La Educación: Retos y Perspectivas." Propósitos y Representaciones 5: XVI, 520 p.-XVI, 520 p. https://www.google.com/books?hl=pt-

$P T \& \mid r=\& i d=A e N e A g A A Q B A J \& o i=f n d \& p g=P P 1 \& d q=\% 22$ Composite + joints+and + connections + principles,$+m o$ delling+and+testing\%22\&ots=XOWk3DvFtv\&sig=MLxosxW8kNDKx2aU2WDZpmEMOFU.

Ministerio de Educación Nacional. 2018. “Protocolo de Accesibilidad Sector Educación.” 57(57).

Monedero,J Cebrián Robles,D. and Desenne, P . 2015. "Usabilidad y Satisfacción En Herramientas de Anotaciones Multimedia Para MOOC." Comunicar: Revista científica iberoamericana de comunicación y educación (44): 55-62.

Moreno, L. , González-García,M. Martínez, P and González, Y. 2017. "Checklist for the Accessible Media Player Evaluation." ASSETS 2017 - Proceedings of the 19th International ACM SIGACCESS Conference on Computers and Accessibility (October): 367-68.

Muñoz, J. Espiñeira-Bellón, E. and Rebollo-Quintela, N. 2016. "Las Píldoras Formativas: Diseño y Desarrollo de Un Modelo de Evaluación En El Espacio Europeo de Educación Superior." Revista de investigación en educación 2(14): 156-69.

Oliveira, B. and Lopes da Silva, A. 2019. "Audiodescrição: Acessibilidade Para Cursos EaD." Revista Brasileira de Aprendizagem Aberta e a Distância 18(1): 15. 
Pagnoni, V. and Mariño, S. 2019. "Calidad de Contenidos En Dominios de Educación. Evaluación de La Accesibilidad Web Mediada Por Validadores Automáticos.” Edmetic 8(1).

Peñafiel, M. and Luján-Mora, S. 2014. "Legislación Sobre Accesibilidad Web: Una Comparativa de Seis Países." 34(2).

Pérez, Lucía. Herranz, M. Abadín, D. and Delgado Santos, C. 2016. "Tecnología de Apoyo y Accesibilidad Cognitiva: De La Autonomía a La Participación." Tecnología y Comunicación. http://riberdis.cedd.net/handle/11181/5165.

Piedra, N et al. 2013. "Producción y Reuso de Recursos Educativos Accesibles." IV Congreso Iberoamericano sobre Calidad y Accesibilidad de la Formación Virtual CAFVIR 2013 (September 2015): 65-79. https://issuu.com/esvial/docs/libroactascafvir2013.

Ramírez Ochoa, M. 2016. "Posibilidades Del Uso Educativo de YouTube." Ra Ximhai: 537-46.

Ruiz, A. Arciniegas, J. and Giraldo, W. 2018. "Caracterización de Marcos de Desarrollo de La Interfaz de Usuario Para Sistemas Interactivos Basados En Distribución de Contenido de Video." Ingeniare. Revista chilena de ingeniería 26(2): 339-53.

Sanchez Gordon, S. , and Luján Mora, S. 2015. “Adaptación de La Metodología de Evaluación de Conformidad Con La Accesibilidad Web WCAG-EM Para Ambientes de e-Learning." Revista Politécnica (November).

Esta obra está bajo una Licencia Creative Commons Attribución-NoCommercial 4.0 International

(cc) BY-NC 